\title{
ITEM INFORMATION AND DISCRIMINATION FUNCTIONS FOR TRINARY PCM ITEMS
}

\author{
WIES AKKERMANS \\ UNIVERSITY OF TWENTE \\ EIJI MURAKI \\ EDUCATIONAL TESTING SERVICE
}

\begin{abstract}
For trinary partial credit items the shape of the item information and the item discrimination function is examined in relation to the item parameters. In particular, it is shown that these functions are unimodal if $\delta_{2}-\delta_{1}<4 \ln 2$ and bimodal otherwise. The locations and values of the maxima are derived. Furthermore, it is demonstrated that the value of the maximum is decreasing in $\delta_{2}-\delta_{1}$. Consequently, the maximum of a unimodal item information function is always larger than the maximum of a bimodal one, and similarly for the item discrimination function.
\end{abstract}

Key words: partial credit model, trinary items, item information function, item discrimination function, maximum item information.

\section{Introduction and Definitions}

Let a graded item admit a score $X$ in $0,1 \ldots$ M. A higher score indicates a better performance. Examinee ability will be denoted by $\theta$. The category response function (CRF) gives the probability of obtaining a score $k$, as a function of $\theta$. CRFs will be denoted by the symbol $\mathrm{P}_{k}(\theta)$ :

$$
\mathrm{P}_{k}(\theta)=\operatorname{Pr}(X=k ; \theta), \quad \text { for } k=0,1 \ldots \mathrm{M} .
$$

In the partial credit model (PCM; Andrich, 1978; Masters, 1982) the CRFs are given by

$$
\mathrm{P}_{k}(\theta)=\frac{\exp \sum_{p=0}^{k}\left(\theta-\delta_{p}\right)}{\sum_{r=0}^{\mathrm{M}}\left[\exp \sum_{p=0}^{r}\left(\theta-\delta_{p}\right)\right]},
$$

with $\Sigma_{p=0}^{0}\left(\theta-\delta_{p}\right) \equiv 0$. The parameters $\delta_{p}$ are the scale values at which two consecutive CRFs intersect. The model for binary items formulated by Rasch (1960) can be seen as a special case of the PCM: the binary Rasch item is a PCM item with $M=1$.

The item response function (IRF) is the (normalized) expected score as a function of $\theta$ :

$$
\operatorname{IRF}(\theta)=\frac{1}{\mathrm{M}} \sum_{k=0}^{\mathrm{M}} k \mathrm{P}_{k}(\theta) .
$$

Requests for reprints should be sent to W. Akkermans, University of Twente, Department of Education, P.O. Box 217, 7500 AE ENSCHEDE, THE NETHERLANDS.

The work reported herein was partially supported under the National Assessment of Educational Progress (Grant No. R999G30002; CFDA No. 84.999G) as administered by the Office of Educational Research and Improvement, US Department of Education. 
The derivative of the IRF is known as the item discrimination function; in this paper it will be denoted by the symbol $\mathrm{G}(\theta)$ :

$$
\mathrm{G}(\theta)=\frac{\partial}{\partial \theta} \operatorname{IRF}(\theta)
$$

Using the fact that in the PCM the derivatives of the CRFs are given by

$$
\frac{\partial}{\partial \theta} \mathrm{P}_{k}(\theta)=\mathrm{P}_{k}(\theta)\left[k-\sum_{r=0}^{\mathrm{M}} r \mathrm{P}_{r}(\theta)\right],
$$

in this model the derivative of the IRF is equal to

$$
\mathrm{G}(\theta)=\frac{1}{\mathrm{M}}\left\{\sum_{k=0}^{\mathrm{M}} k^{2} \mathrm{P}_{k}(\theta)-\left[\sum_{k=0}^{\mathrm{M}} k \mathrm{P}_{k}(\theta)\right]^{2}\right\} .
$$

Let $\mathrm{L}(\theta \mid X)$ be the likelihood function of $\theta$ given the observed response $X$. Again using (2), the item information function $\mathrm{I}(\theta)=E\left\{-\partial^{2} / \partial \theta^{2}[\ln L(\theta \mid X) ; \theta]\right\}$ follows as

$$
\begin{aligned}
\mathrm{I}(\theta) & =\sum_{k=0}^{M}\left[\frac{\partial}{\partial \theta} \mathrm{P}_{k}(\theta)\right]^{2} / \mathrm{P}_{k}(\theta) \\
& =\sum_{k=0}^{M} k^{2} \mathrm{P}_{k}(\theta)-\left[\sum_{k=0}^{\mathrm{M}} k \mathrm{P}_{k}(\theta)\right]^{2} .
\end{aligned}
$$

Note that in the PCM the functions $\mathrm{I}(\theta)$ and $\mathrm{G}(\theta)$ are proportional. Let a trinary item be a graded item with maximum score 2 . Huynh (1994) shows that $\delta_{2}-\delta_{1} \geq 2 \ln 2$ is a necessary and sufficient condition for the likelihood of the score on a trinary partial credit item to be fully equivalent to the likelihood of the total score on a set of 2 independent binary Rasch items. Subsequently, Huynh introduces the term "indecomposable" (Huynh, 1996) to refer to trinary PCM items with $\delta_{2}-\delta_{1}<2 \ln 2$; and he proves that the likelihood of the score on any PCM item is equivalent to the likelihood of the total score on a set of independent binary and indecomposable trinary PCM items. Huynh also uses the term trinary Rasch item for the trinary PCM item.

Binary Rasch items have been thoroughly investigated, their features are well known. For example, the information function of a binary Rasch item is unimodal, its maximum occurs at $\theta=\delta$, and the value of the maximum is equal to $1 / 4$. It is also well known that in the Rasch model the value of the derivative of the IRF, evaluated at $\theta=\delta$, equals $1 / 4$. Much less appears to be known about the characteristics of trinary Rasch (PCM) items. Thus, as the PCM appears to be built up of both binary and trinary Rasch items, there seems to be a need for investigating the trinary Rasch item.

This paper will concentrate on the item information function $I(\theta)$ and the item discrimination function $\mathrm{G}(\theta)$ of the trinary PCM item. The conditions will be derived under which these functions have either one or two modes, and the locations and values of the maxima will be determined. Because of the proportionality of $\mathrm{I}(\theta)$ and $\mathrm{G}(\theta)$ in this model, the calculations will be carried out for $\mathrm{I}(\theta)$ only.

\section{Condition for Unimodality}

Let $\operatorname{Var}(X ; \theta)$ be the variance of $X$ as a function of $\theta$. Because $\operatorname{Var}(X ; \theta)=$ $\sum_{k=0}^{\mathrm{M}} k^{2} \mathrm{P}_{k}(\theta)-\left[\sum_{k=0}^{\mathrm{M}} k \mathrm{P}_{k}(\theta)\right]^{2}$, it appears that for the $\mathrm{PCM} \operatorname{Var}(X ; \theta)$ is equal to $\mathrm{I}(\theta)$, as given in (3). As it is well known that $\operatorname{Var}(X ; \theta)$ approaches 0 for $\theta \rightarrow \pm \infty$, it follows that also 


$$
\lim _{\theta \rightarrow \infty} \mathrm{I}(\theta)=\lim _{\theta \rightarrow-\infty} \mathrm{I}(\theta)=0
$$

Defining $\bar{\delta}=\left(\delta_{1}+\delta_{2}\right) / 2$, it is easy to show that for a trinary PCM item,

$$
\mathrm{I}(\theta) \text { is symmetric around } \theta=\bar{\delta} \text {. }
$$

Starting from (3) and once more using (2), the derivative of the item information function can be found; it is equal to

$$
\frac{\partial}{\partial \theta} \mathrm{I}(\theta)=\sum_{k=0}^{\mathrm{M}} k^{3} \mathrm{P}_{k}(\theta)-3\left[\sum_{k=0}^{\mathrm{M}} k \mathrm{P}_{k}(\theta)\right] \sum_{k=0}^{\mathrm{M}} k^{2} \mathrm{P}_{k}(\theta)+2\left[\sum_{k=0}^{\mathrm{M}} k \mathrm{P}_{k}(\theta)\right]^{3}
$$

If we let $\xi=\exp (\theta), \varepsilon_{1}=\exp \left(-\delta_{1}\right), \varepsilon_{2}=\exp \left(-\delta_{2}\right)$ and substitute these into the expressions for $\mathrm{P}_{k}(\theta)$, then after some algebra and rearranging this derivative can be expressed as

$$
\frac{\partial}{\partial \theta} \mathrm{I}(\theta)=\xi \varepsilon_{1} \frac{\left(1-\xi^{2} \varepsilon_{1} \varepsilon_{2}\right)\left[\xi^{2} \varepsilon_{1} \varepsilon_{2}-\xi\left(\varepsilon_{1}-8 \varepsilon_{2}\right)+1\right]}{\left(1+\xi \varepsilon_{1}+\xi^{2} \varepsilon_{1} \varepsilon_{2}\right)^{3}} .
$$

This function becomes or approaches zero in each of the following cases:

$$
\begin{array}{ll}
\text { if } \xi \rightarrow \infty, & \text { i.e., if } \theta \rightarrow \infty, \\
\text { if } \xi \rightarrow 0, & \text { i.e., if } \theta \rightarrow-\infty, \\
\text { if } \xi^{2}=1 /\left(\varepsilon_{1} \varepsilon_{2}\right), & \text { i.e., if } \theta=\bar{\delta}, \\
\text { if } \xi^{2} \varepsilon_{1} \varepsilon_{2}-\xi\left(\varepsilon_{1}-8 \varepsilon_{2}\right)+1=0 .
\end{array}
$$

To start with, therefore, there always exist one finite and two asymptotic solutions to $\partial / \partial \theta[\mathrm{I}(\theta)]=0$. The number of solutions to $\partial / \partial \theta \mathrm{I}(\theta)=0$ furthermore depends upon the discriminant $D=\left(\varepsilon_{1}-8 \varepsilon_{2}\right)^{2}-4 \varepsilon_{1} \varepsilon_{2}$ of the quadratic in (8). This quadratic has two real solutions if its discriminant is positive, that is, if

$$
\frac{\varepsilon_{1}}{\varepsilon_{2}} \leq 4 \text { or } \quad \frac{\varepsilon_{1}}{\varepsilon_{2}} \geq 16
$$

Let the real solutions to this quadratic, if they exist, be denoted by $\xi_{1}$ and $\xi_{2}$. In order for these solutions to be valid they both have to be positive, as $\xi=\exp (\theta)>0$. A necessary and sufficient condition for two real numbers to be both positive is that both their sum and their product be positive. The sum of the two roots to the quadratic $p x^{2}+q x+r$ equals $-q / p$, and their product is $r / p$; therefore we need $\left(\varepsilon_{1}-8 \varepsilon_{2}\right) / \varepsilon_{1} \varepsilon_{2}>0$ and $1 / \varepsilon_{1} \varepsilon_{2}>0$. The latter condition poses no problem; the former is fulfilled if $\varepsilon_{1} / \varepsilon_{2}>8$. Hence, if there are two real roots $\xi_{1}, \xi_{2}$ to the quadratic in (8), these can only both be positive if

$$
\frac{\varepsilon_{1}}{\varepsilon_{2}}>8
$$

Because of (10) only the second possibility in (9) is useful. Remembering that $\varepsilon_{1} / \varepsilon_{2}=$ $\exp \left(\delta_{2}-\delta_{1}\right)$ it may be concluded that the quadratic in (8) has

$$
\begin{array}{ll}
\text { no solutions } & \text { if } \delta_{2}-\delta_{1}<4 \ln 2, \\
\text { two solutions } & \text { if } \delta_{2}-\delta_{1} \geq 4 \ln 2 .
\end{array}
$$

In the first case, the information function will only have the one finite and two asymptotic extremes derived in (5) through (7) above, that is, at $\theta=\bar{\delta}$ and for $\theta \rightarrow \pm \infty$; in the second 
case there are two more extremes in the information function. These two cases will be examined separately below.

\section{Unimodal Item Information Function}

Using (5) through (7), the symmetry of $I(\theta)$, Equation (4), and the fact that the information function is always positive, it may be concluded that if there is only one finite extreme in the information function this function has to be unimodal with a maximum occurring at $\theta=\bar{\delta}$. In order to find the value of this single maximum $\mathrm{I}(\bar{\delta})$ note that $\bar{\delta}-\delta_{1}=\left(\delta_{2}-\delta_{1}\right) / 2$ and that $\exp \left(2 \bar{\delta}-\delta_{1}-\delta_{2}\right)=1$, so that, using (3) and (1), it can be verified that

$$
I(\bar{\delta})=\frac{2}{2+\exp \left[\left(\delta_{2}-\delta_{1}\right) / 2\right]}
$$

From (11) it follows that

$$
\mathrm{I}(\bar{\delta}) \text { is decreasing in } \delta_{2}-\delta_{1} .
$$

The maximum of the information at $\theta=\bar{\delta}$ therefore occurs if $\delta_{2}-\delta_{1} \rightarrow-\infty$, that is, if $\delta_{2} \ll \delta_{1}$; and the minimum of the information at $\theta=\bar{\delta}$ is reached if $\delta_{2}-\delta_{1} \rightarrow \infty$, that is, when $\delta_{2} \gg \delta_{1}$. However, for $\delta_{2}-\delta_{1} \geq 4 \ln 2$ the information function is no longer single peaked: hence the minimum value of the maximum at $\theta=\bar{\delta}$ for a single peaked information function occurs for $\delta_{2}-\delta_{1}=4 \ln 2$. Using (11), values for the maximum at $\theta=\bar{\delta}$ may be easily obtained; some values for the maximum of a unimodal information function are:

$$
\begin{array}{lll}
\mathrm{I}(\bar{\delta}) \approx 1 & \text { for } & \delta_{2} \ll \delta_{1}, \\
\mathrm{I}(\bar{\delta})=\frac{2}{3} & \text { for } & \delta_{1}=\delta_{2}, \\
\mathrm{I}(\bar{\delta})=\frac{1}{2} & \text { for } & \delta_{2}-\delta_{1}=2 \ln 2, \\
\mathrm{I}(\bar{\delta})=\frac{1}{3} & \text { for } & \delta_{2}-\delta_{1}=4 \ln 2 .
\end{array}
$$

From (12) and the fact that for $\delta_{2}-\delta_{1} \geq 4 \ln 2$ the item information function is no longer unimodal, it follows that the value of the single maximum at $\theta=\bar{\delta}$ is bounded by 1 and $1 / 3$.

\section{Bimodal Item Information Function}

If the quadratic in (8) has two real roots, then because of the symmetry of $I(\theta)$ there must be maxima at these roots and a minimum in between, that is, at $\theta=\bar{\delta}$. Solving the quadratic it follows that the two maxima will occur at $\xi_{1,2}=\left\{\varepsilon_{1}-8 \varepsilon_{2} \pm\left[\left(\varepsilon_{1}-8 \varepsilon_{2}\right)^{2}-\right.\right.$ $\left.\left.4 \varepsilon_{1} \varepsilon_{2}\right]^{1 / 2}\right\} / 2 \varepsilon_{1} \varepsilon_{2}$. Remembering that $\xi=\exp (\theta), \varepsilon_{1}=\exp \left(-\delta_{1}\right)$ and $\varepsilon_{2}=\exp \left(-\delta_{2}\right)$, this can be rewritten as

$$
\theta_{1,2}=\bar{\delta} \pm \ln \left\{\frac{\varepsilon_{1}-8 \varepsilon_{2}+\left[\left(\varepsilon_{1}-8 \varepsilon_{2}\right)^{2}-4 \varepsilon_{1} \varepsilon_{2}\right]^{1 / 2}}{2\left(\varepsilon_{1} \varepsilon_{2}\right)^{1 / 2}}\right\} .
$$

In the appendix it is shown that the value of these maxima is equal to

$$
\mathrm{I}\left(\theta_{1}\right)=\mathrm{I}\left(\theta_{2}\right)=\frac{1}{4\left\{1-4 \exp \left[-\left(\delta_{2}-\delta_{1}\right)\right]\right\}} .
$$


Again,

$$
\mathrm{I}\left(\theta_{1}\right) \text { and } \mathrm{I}\left(\theta_{2}\right) \text { are decreasing in } \delta_{2}-\delta_{1} .
$$

For $\delta_{2}-\delta_{1}=4 \ln 2$, both maxima still are located at $\theta=\bar{\delta}$, and their value is $1 / 3$. For $\delta_{2} \gg \delta_{1}$, the maxima will be located near $\delta_{2}$ and $\delta_{1}$, respectively, as can be seen upon taking the limit of (17) for $\delta_{2}-\delta_{1} \rightarrow \infty$, that is, for $\varepsilon_{2} / \varepsilon_{1} \rightarrow 0$. In this case the exponential $\exp \left[-\left(\delta_{2}-\delta_{1}\right)\right]$ will approach 0 and hence

$$
\mathrm{I}\left(\theta_{1}\right) \approx \mathrm{I}\left(\delta_{1}\right) \approx \frac{1}{4} \text { for } \delta_{2} \gg \delta_{1},
$$

and similarly for the maximum located near $\delta_{2}$. It may be concluded that the values of the maxima of a bimodal information function are bounded by $1 / 3$ and $1 / 4$.

As an example of a trinary item, let $\left(\delta_{1}, \delta_{2}\right)=(-2,2)$. For this item the maxima will be located at $\theta= \pm 1.8154$, and their value is .270 . An item with $\left(\delta_{1}, \delta_{2}\right)=(-3,3)$ has its maxima at $\theta= \pm 2.9974$, and their value is .252 . Note that indeed this value is nearly equal to $1 / 4$.

\section{Item Discrimination Function}

With the appropriate modifications, all the above holds for the item discrimination function $G(\theta)$ as well. In particular, $G(\theta)$ will be bi- or unimodal under the same conditions, and the maxima will have the same location. Their values are obtained upon dividing (11) and (18) by $M$, which in this case is equal to 2 .

If $G(\theta)$ has one maximum, the second derivative of the item response function changes sign exactly once: the IRF then has one point of inflection. A function with only one point of inflection is smooth. Hence the IRF of a PCM item with $\delta_{2}-\delta_{1}<4 \ln 2$ will be smooth. In fact, its smoothness is comparable to the smoothness of a Rasch function. If $\mathrm{G}(\theta)$ is bimodal, there will be a "bump" in the middle of the corresponding IRF.

\section{Some Comparisons}

The maximum information of a single binary Rasch item equals $1 / 4$. Therefore the information obtainable with the total score on 2 independent binary Rasch items can never exceed 1/2. Bearing this in mind, and also the values given in (13) through (16) and (20), some remarks apply:

1. For trinary PCM items, the value of the maximum information is decreasing in $\delta_{2}-\delta_{1}$. This follows from the fact that these maxima are, first, decreasing in $\delta_{2}-\delta_{1}$ for both unimodal and bimodal information functions (see equations 12 and 19), and, second, equal for $\delta_{2}-\delta_{1}=4 \ln 2$.

2. Hence, for trinary PCM items the maximum of every unimodal information function is larger than the maximum of every bimodal information function.

3. By a similar argument, the maximum information of every indecomposable trinary PCM item is larger than the maximum information of every decomposable trinary PCM item.

4. Consequently, the maximum information of every indecomposable trinary PCM item is larger than the maximum information obtainable with 2 independent binary Rasch items. Therefore indecomposable trinary PCM items are in a sense more efficient than the total score on two independent binary Rasch items can ever be.

5. For trinary PCM items with $\delta_{2} \geq \delta_{1}$, the maximum information never exceeds $2 / 3$.

6. If $\delta_{2} \gg \delta_{1}$, the maximum information obtainable with a trinary PCM item is equal to the maximum information for just a single binary Rasch item. PCM items with $\delta_{2} \gg \delta_{1}$ are perhaps best understood upon examining their expected score distributions. Assuming that $\delta_{2} \gg \delta_{1}$ implies both $\bar{\delta} \gg \delta_{1}$ and $\delta_{2} \gg \bar{\delta}$, then for $\theta \ll$ $\bar{\delta}$ the probability of obtaining a score 2 is nearly 0 , and for these values of $\theta$ the 
PCM item behaves as a binary Rasch item with parameter approximately equal to $\delta_{1}$. For $\theta \gg \bar{\delta}$, the probability of obtaining a score 0 will be nearly 0 , and for these values of $\theta$ the PCM item behaves as a binary Rasch item with parameter approximately equal to $\delta_{2}$. For "average" values of $\theta$, which may be quite a substantial part of the $\theta$-axis if $\delta_{2}$ really is much larger than $\delta_{1}$, the probability of obtaining a score 1 on the PCM item will be nearly equal to 1 . So in the limit, for $\delta_{2} \gg \delta_{1}$, the decomposable trinary Rasch item is equivalent to two independent binary Rasch items with parameters approximately equal to $\delta_{1}$ and $\delta_{2}$. Its information function will resemble the information function of the total score on two widely separated Rasch items, with information approximately equal to zero for $\theta$ in the neighbourhood of $\bar{\delta}$.

7. The other limit, that is, $\delta_{2} \ll \delta_{1}$, is also interesting. Note that $\mathrm{P}_{1}(\theta)$ reaches its maximum at $\theta=\bar{\delta}$. Now if $\delta_{2} \ll \delta_{1}$, the maximum $\mathrm{P}_{1}(\bar{\delta}) \approx 0$, and hence $\mathrm{P}_{1}(\theta) \approx$ 0 for all $\theta$. Consequently, in this case $\mathrm{P}_{0}(\theta)+\mathrm{P}_{2}(\theta) \approx 1$ for all values of $\theta$; and because $\mathrm{P}_{0}(\theta) / \mathrm{P}_{2}(\theta)=1 /\{\exp [2(\theta-\bar{\delta})]\}$, it follows that $\mathrm{P}_{0}(\theta) \approx 1 /\{1+\exp [2(\theta-$ $\bar{\delta})]\}$ and $\mathrm{P}_{2}(\theta) \approx \exp [2(\theta-\bar{\delta})] /\{1+\exp [2(\theta-\bar{\delta})]\}$. So for $\delta_{2} \ll \delta_{1}$ the PCM item reduces to a 2 parameter logistic item (Birnbaum, 1968) with location parameter $\bar{\delta}$ and discrimination equal to 2 . The maximum information for such an item is equal to 1 (see next section).

\section{Discrimination Parameter}

Under the generalized partial credit model (GPCM; see Muraki, 1992; Muraki, 1993), the category response functions are given by

$$
\mathrm{P}_{k}(\theta)=\frac{\exp \left[\alpha \sum_{p=0}^{k}\left(\theta-\delta_{p}\right)\right]}{\sum_{r=0}^{\mathrm{M}} \exp \left[\alpha \sum_{p=0}^{r}\left(\theta-\delta_{p}\right)\right]},
$$

again with $\Sigma_{p=0}^{0}\left(\theta-\delta_{p}\right) \equiv 0$. In this model the discrimination parameter $\alpha$ varies over items. If in this case we let $\xi=\exp (\alpha \theta), \varepsilon_{1}=\exp \left(-\alpha \delta_{1}\right)$ and $\varepsilon_{2}=\exp \left(-\alpha \delta_{2}\right)$, all derivations will be analogous to the ones given in the previous sections, resulting in unimodal item information and discrimination functions if $\alpha\left(\delta_{2}-\delta_{1}\right)<4 \ln 2$ and bimodal functions otherwise. The values of the item information and discrimination are now equal to $\alpha^{2} \operatorname{Var}(X ; \theta)$ and $(\alpha / \mathrm{M}) \operatorname{Var}(X ; \theta)$, respectively. The single maximum is also located at $\theta=\bar{\delta}$; the location of the bimodal maximum can be adapted from (17):

$$
\theta_{1,2}=\bar{\delta} \pm \frac{1}{\alpha} \ln \left\{\frac{\varepsilon_{1}-8 \varepsilon_{2}+\left[\left(\varepsilon_{1}-8 \varepsilon_{2}\right)^{2}-4 \varepsilon_{1} \varepsilon_{2}\right]^{1 / 2}}{2\left(\varepsilon_{1} \varepsilon_{2}\right)^{1 / 2}}\right\} .
$$

Furthermore, note that the number $4 \ln 2$ is invariant under linear transformation of the $\theta$-scale: setting $\theta^{*}=k \theta+m$ and offsetting this in the usual way by $\alpha^{*}=\alpha / k$ and $\delta^{*}=$ $k \delta+m$ will yield

$$
\frac{\varepsilon_{1}^{*}}{\varepsilon_{2}^{*}}=\frac{\exp \left(-\alpha^{*} \delta_{1}^{*}\right)}{\exp \left(-\alpha^{*} \delta_{2}^{*}\right)}=\frac{\exp \left[-\frac{\alpha}{k}\left(k \delta_{1}+m\right)\right]}{\exp \left[-\frac{\alpha}{k}\left(k \delta_{2}+m\right)\right]}=\frac{\exp \left(-\alpha \delta_{1}\right)}{\exp \left(-\alpha \delta_{2}\right)}=\frac{\varepsilon_{1}}{\varepsilon_{2}} .
$$

Hence in any linearly transformed metric $\theta^{*}, \mathbf{I}\left(\theta^{*}\right)$ will be unimodal if $\alpha^{*}\left(\delta_{2}^{*}-\delta_{1}^{*}\right)<4 \ln$ 2 , and bimodal otherwise. 


\section{Conclusion and Discussion}

The necessary and sufficient conditions have been stated under which both the item information and the item discrimination function of a trinary PCM item are unimodal, and the location and value of these maxima were derived. It was furthermore ascertained that for trinary PCM items the maximum of a unimodal item information function is always larger than the maximum of a bimodal one; and similarly for the item discrimination function. As a consequence, the IRF of a trinary PCM item with a unimodal discrimination function is both steeper and smoother than the IRF of a trinary PCM item with a bimodal discrimination function. The smoothness of the former is comparable to that of a binary Rasch item. Although the condition for bimodality of the item information function seems rather strong $\left(\delta_{2}-\delta_{1}>4 \ln 2=2.77\right)$, these items do occur in practice. For example, in the 1994 survey of the National Assessment of Educational Progress (NAEP), students of age 13 were administered 449 items covering Geometry, History and Reading. Of these items, 86 were trinary PCM items, and 11 of them had a bimodal information function. This is about 13 percent. At the time of writing, the analyses of the 1994 NAEP data is still in progress; however, a general overview of the scaling procedures used can be found in Mislevy, Johnson \& Muraki (1992).

The results obtained in this paper may have some practical relevance. Matching a target information function is a commonly used criterion in test design. This may require, at some point in the test construction process, finding an item with a pre-specified information at a certain theta level, say at $\theta_{0}$. For binary Rasch items this is easy: under the Rasch model item information functions differ only in their location, and hence the item located closest to $\theta_{0}$ will have higher information at $\theta_{0}$ than all other items. For polytomous items, however, finding the best item is no trivial task. In order to avoid having to calculate $\mathrm{I}\left(\theta_{0}\right)$ for many items, knowledge of the location and value of the maxima might be helpful. At least for trinary PCM items these are now available.

Another practical question is that of the optimal value of $M$. Assume an infinite item pool, and consider a situation in which an item with high information at $\theta=\theta_{0}$ is required. As an example, one might think of computerized adaptive testing, where a provisional ability estimate $\hat{\theta}^{(t)}=\theta_{0}$ is available. From an infinite item pool it will be possible to select an indecomposable trinary PCM item, with its maximum information located at $\theta=\theta_{0}$. As has been demonstrated, the maximum information of an indecomposable trinary PCM item is larger than the maximum information obtainable with any two independent Rasch items. Theoretically, therefore, (infinite item pools do not exist), in this case administration of a suitable, that is, indecomposable trinary item is more efficient than administration of two independent binary Rasch items can ever be. This does not generalize to $M>2$ : the likelihood of every PCM item with $M>2$ is equivalent to the likelihood of the total score on a number of independent binary and indecomposable trinary PCM items, and therefore the two information functions will be equal. It follows that, even in an infinite item pool, there exist no $\mathrm{PCM}$ items with $\mathrm{M}>2$, having larger maximum information at $\theta=\theta_{0}$, than would be obtainable with the equivalent combination of independent binary and trinary PCM items.

This may have implications for test construction and item banking. It could be argued that the construction of more items with small $M$ (i.e., $M=1$ and $M=2$ ) might be more profitable than the construction of less items, each of these with large M. First, in the infinite item pool, for every PCM item with $M>2$ there will exist an equivalent combination of independent binary and trinary PCM items having the same information function. Second, from a practical perspective, there will be more items available to choose from. Third, the maximum information for items with $\mathrm{M} \leq 2$ can now be calculated and hence these items may become more easily manageable in item banks than items with large $M$. It should be 
remarked, however, that not all trinary items are equally efficient: in particular, those with $\delta_{2}$ much larger than $\delta_{1}$ have approximately zero information for average values of $\theta$.

The value of the maximum information is decreasing in $\delta_{2}-\delta_{1}$. This would seem to suggest that it is desirable to have items with $\delta_{2}$ as small as possible, compared to the value of $\delta_{1}$. However, for rating scale items it might be inappropriate to have $\delta_{2}<\delta_{1}$ (Andrich, 1982). Furthermore, although, of all trinary PCM items, the maximum information of items with $\delta_{2} \ll \delta_{1}$ is largest, it was pointed out that these items do not really behave as trinary items: they are equivalent to binary 2 parameter logistic items with $\alpha=2$.

Finally, in the context of multiple choice items there are several reasons for arguing that the optimal number of choices be 3 (e.g. Lord, 1980). Some of these reasons have to do with discrimination and reliability. It would be interesting to examine whether there is any relationship with 3 seeming the most efficient number of categories for graded items, too.

\section{Appendix}

Calculation of the maxima for a bimodal information function

In this appendix three shorthand symbols will be used:

$$
\begin{gathered}
\mathrm{f}(\theta)=\exp \left(\theta-\delta_{1}\right), \\
\mathrm{g}(\theta)=\exp \left(2 \theta-\delta_{1}-\delta_{2}\right), \\
y=\exp \left(\delta_{2}-\delta_{1}\right) .
\end{gathered}
$$

Note that $\mathrm{g}(\theta)$ can be expressed in $\mathrm{f}(\theta)$ and $y$ :

$$
\mathrm{g}(\theta)=[\mathrm{f}(\theta)]^{2} / y \text {. }
$$

Using (3) and the shorthand notations $\mathrm{f}(\theta)$ and $\mathrm{g}(\theta)$, for a trinary PCM item $\mathrm{I}(\theta)=\mathrm{P}_{1}(\theta)$ $+4 \mathrm{P}_{2}(\theta)-\left[\mathrm{P}_{1}(\theta)+2 \mathrm{P}_{2}(\theta)\right]^{2}$ can be written as

$$
\begin{aligned}
\mathrm{I}(\theta) & =\mathrm{P}_{1}(\theta)\left[1-\mathrm{P}_{1}(\theta)\right]+4 \mathrm{P}_{2}(\theta)\left[1-\mathrm{P}_{2}(\theta)\right]-4 \mathrm{P}_{1}(\theta) \mathrm{P}_{2}(\theta) \\
& =\frac{\mathrm{f}(\theta)[1+\mathrm{g}(\theta)]+4 \mathrm{~g}(\theta)[1+\mathrm{f}(\theta)]-4 \mathrm{f}(\theta) \mathrm{g}(\theta)}{[1+\mathrm{f}(\theta)+\mathrm{g}(\theta)]^{2}} \\
& =\frac{f(\theta)+\mathrm{f}(\theta) \mathrm{g}(\theta)+4 \mathrm{~g}(\theta)}{[1+\mathrm{f}(\theta)+\mathrm{g}(\theta)]^{2}} .
\end{aligned}
$$

Substituting (21) into (22) it is possible to express $\mathrm{I}(\theta)$ in $\mathrm{f}(\theta)$ and $y$ only:

$$
\begin{aligned}
\mathrm{I}(\theta) & =\frac{\mathrm{f}(\theta)+\mathrm{f}(\theta)[\mathrm{f}(\theta)]^{2} / y+4[\mathrm{f}(\theta)]^{2} / y}{\left\{1+\mathrm{f}(\theta)+[\mathrm{f}(\theta)]^{2} / y\right\}^{2}} \\
& =\frac{y \mathrm{f}(\theta)\left\{y+[\mathrm{f}(\theta)]^{2}+4 \mathrm{f}(\theta)\right\}}{\left\{y+y \mathrm{f}(\theta)+[\mathrm{f}(\theta)]^{2}\right\}^{2}}
\end{aligned}
$$

For a bimodal information function the locations of the maxima, expressed in $\xi=\exp (\theta)$ and $\varepsilon_{k}=\exp \left(-\delta_{k}\right)$, may be rewritten from the expression just above (17):

$$
\xi_{1,2}=\frac{1}{2 \varepsilon_{1}}\left\{\frac{\varepsilon_{1}}{\varepsilon_{2}}-8 \pm\left[\left(\frac{\varepsilon_{1}}{\varepsilon_{2}}-8\right)^{2}-4 \frac{\varepsilon_{1}}{\varepsilon_{2}}\right]^{1 / 2}\right\} .
$$


Noting that $\varepsilon_{1} / \varepsilon_{2}=\exp \left(\delta_{2}-\delta_{1}\right)=y$, the locations of the maxima expressed in $\theta$ are given by

$$
\theta_{1,2}=\ln \left\{\frac{1}{2 \exp \left(-\delta_{1}\right)}\left\{y-8 \pm\left[(y-8)^{2}-4 y\right]^{1 / 2}\right\}\right\} .
$$

Concentrate for the moment on the maximum located at $\theta_{1}$. Evaluating $\mathrm{f}(\theta)$ for $\theta=\theta_{1}$ will give, with $\mathrm{f}\left(\theta_{1}\right)=\exp \left(\theta_{1}-\delta_{1}\right)$ :

$$
f\left(\theta_{1}\right)=\frac{1}{2}\left\{y-8+\left[(y-8)^{2}-4 y\right]^{1 / 2}\right\} .
$$

Instead of directly trying to evaluate $\mathrm{I}(\theta)$ at $\theta=\theta_{1}$, note that with the help of (24) it is possible to express $\left[\mathrm{f}\left(\theta_{1}\right)\right]^{2}$ in $\mathrm{f}\left(\theta_{1}\right)$ and $y$ :

$$
\begin{aligned}
{\left[\mathrm{f}\left(\theta_{1}\right)\right]^{2} } & =\frac{1}{4}\left\{(y-8)^{2}+2(y-8)\left[(y-8)^{2}-4 y\right]^{1 / 2}+(y-8)^{2}-4 y\right\} \\
& =\frac{1}{2}(y-8)\left\{y-8+\left[(y-8)^{2}-4 y\right]^{1 / 2}\right\}-y \\
& =(y-8) \mathbf{f}\left(\theta_{1}\right)-y .
\end{aligned}
$$

Now in order to evaluate $\mathrm{I}(\theta)$ at $\theta=\theta_{1}$, first, in (23), substitute $\left[\mathrm{f}\left(\theta_{1}\right)\right]^{2}$ by the expression derived in (25):

$$
\begin{aligned}
\mathbf{I}\left(\theta_{1}\right) & =\frac{y \mathrm{f}\left(\theta_{1}\right)\left[y+(y-8) \mathrm{f}\left(\theta_{1}\right)-y+4 \mathrm{f}\left(\theta_{1}\right)\right]}{\left[y+y \mathrm{f}\left(\theta_{1}\right)+(y-8) \mathrm{f}\left(\theta_{1}\right)-y\right]^{2}} \\
& =\frac{y \mathrm{f}\left(\theta_{1}\right)\left[y \mathrm{f}\left(\theta_{1}\right)-4 \mathrm{f}\left(\theta_{1}\right)\right]}{\left[2 y \mathrm{f}\left(\theta_{1}\right)-8 \mathrm{f}\left(\theta_{1}\right)\right]^{2}} \\
& =\frac{y}{4(y-4)} .
\end{aligned}
$$

For the maximum located at $\theta_{2}$ the derivation is similar. Therefore the value of the maximum of a bimodal information function follows upon replacing $y$ by $\exp \left(\delta_{2}-\delta_{1}\right)$ in the above expression, yielding

$$
I\left(\theta_{1}\right)=\mathbf{I}\left(\theta_{2}\right)=\frac{\exp \left(-\delta_{1}\right)}{4\left[\exp \left(-\delta_{1}\right)-4 \exp \left(-\delta_{2}\right)\right]} .
$$

\section{References}

Andrich, D. (1978). A rating formulation for ordered response categories. Psychometrika, 43, 561-573.

Andrich, D. (1982). An extension of the Rasch model for ratings providing both location and dispersion parameters. Psychometrika, 47, 105-113.

Birnbaum, A. (1968). Some latent trait models and their use in inferring an examinee's ability. In F. M. Lord \& M. R. Novick (Eds.), Statistical theories of mental test scores (pp. 395-479). Reading, MA: Addison-Wesley.

Huynh, H. (1994). On equivalence between a partial credit item and a set of independent Rasch binary items. Psychometrika, 59, 111-119.

Huynh, H. (1996). Decomposition of a Rasch partial credit item into independent binary and indecomposable trinary items. Psychometrika, 61, 31-39.

Lord, F. M. (1980). Applications of item response theory to practical testing problems. Hillsdale, NY: Lawrence Erlbaum.

Masters, G. N. (1982). A Rasch model for partial credit scoring. Psychometrika, 47, 149-174.

Mislevy, R., Johnson, E., \& Muraki, E. (1992). Scaling procedures in NAEP. Jotimal of Educational Statistics, 17 , 131-154. 
Muraki, E. (1992). A generalized partial credit model: Application of an EM algorithm. Applied Psychological Measurement, 16, 159-176.

Muraki, E. (1993). Information functions of the generalized partial credit model. Applied Psychological Measurement, 17, 351-363.

Rasch, G. (1960). Probabilistic models for some intelligence and attainment tests. Copenhagen: Danish Institute for Educational Research. (Reprinted in 1980 by The University of Chicago Press).

Manuscript received 11/9/95

Final version received 10/23/96 